\title{
Thermally induced principal parametric resonance in circular plates
}

\author{
Ali H. Nayfeh and Waleed Faris \\ Department of Engineering Science and Mechanics, MC 0219, Virginia Polytechnic Institute and State University, \\ Blacksburg, Virginia 24061, USA
}

Received 3 July 2001

Revised 29 October 2001

\begin{abstract}
We consider the problem of large-amplitude vibrations of a simply supported circular flat plate subjected to harmonically varying temperature fields arising from an external heat flux (aeroheating for example). The plate is modeled using the von Karman equations. We used the method of multiple scales to determine an approximate solution for the case in which the frequency of the thermal variations is approximately twice the fundamental natural frequency of the plate; that is, the case of principal parametric resonance. The results show that such thermal loads produce large-amplitude vibrations, with associated multi-valued responses and subcritical instabilities.
\end{abstract}

\section{Introduction}

The static and dynamic behavior of flat and curved plates, including isotropic and composite plates, subjected to thermal loads is a problem of considerable relevance in the design and development of supersonic/hypersonic vehicles, reusable space transportation, and launch vehicles [11]. Other applications could be found in thermally actuated composite diaphragms [19], pressure sensors that are used in automotive engines, compensation of thermally induced effects in force resonant sensors [6], and brake discs and rotors. Our interest here is in isotropic plates. The previous works on this subject could be classified into two categories. The first category concentrated on solving the plate equations for given thermal distributions, and the second category formulated the problem as a thermo-elasticity problem.

The first category includes the studies of Heldenfels and Roberts [7] and Gatewood [5] who solved the thermal flat plate problem for small deflections and constant temperature distributions. Buckens [2] examined

*Corresponding author. Tel.: +1 540231 5453; Fax: +1 540231 2299; E-mail: anayfeh@vt.edu. small deflections of rectangular plates under thermal loads and analyzed their buckling. Sunakawa [21] studied the influence of temperature changes on the largeamplitude free vibrations of rectangular plates using successive approximations. He showed that these effects are not negligible. In a series of publications, Pal [16-18] examined the large deformations of thermal circular plates using Berger's approximation. Jones et al. [10] examined the large-amplitudes vibrations and, buckling of a rectangular plate under elevated temperatures using also Berger's approximation and including viscoelastic effects. They showed that the Berger's approximation is the first term in a perturbation expansion of the von Karman equations. Heuer et al. [9] also studied the nonlinear vibrations and buckling of flat plates using Berger's approximation.

The second category includes the study of Marguerre [12] who studied the linear decoupled thermoelastic plate problem. Sokolnikoff and Sokolnikoff [20] studied the linear thermal stresses in clamped elastic plates under constant linear temperature distributions and showed that the solution is similar to the solution of equivalent transverse loaded elastic plates. This point was later elaborated by Boley and Weiner [3]. Chang and Wan [4] solved the thermo-coupled Berger equation for flat-plate vibrations. Altay and Dokmeci [1] formu- 


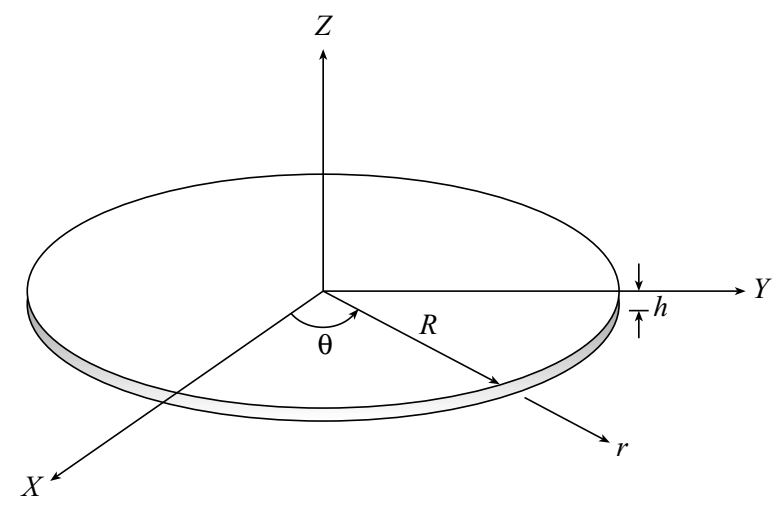

Fig. 1. Geometry of a circular plate.

lated the Karman-Mindlin plate equations for thermoelastic vibrations of temperature-dependent materials.

In this paper, we analyze the nonlinear response of a simply supported circular plate with movable edges to harmonically varying thermal loads. In particular, we consider the case of principal parametric resonance.

\section{Problem formulation}

First, we consider the coupled heat equation and the dynamic version of von Karman equations for a thermally excited circular plate. The basic geometric features of the circular plate are shown in Fig. 1. The thermal loading is assumed to be axisymmetric, and hence the plate vibrations are axisymmetric. They are governed by Chang and Wan [4]

$$
\begin{gathered}
k \nabla^{2} T+Q=\rho c_{p} \frac{\partial T}{\partial t}+\frac{E \alpha T_{0}}{1-2 v} \frac{\partial e}{\partial t} \\
D \nabla^{4} w+\rho h \ddot{w} \\
=\frac{1}{r} \frac{\partial^{2} w}{\partial r^{2}} \frac{\partial F}{\partial r}+\frac{1}{r} \frac{\partial w}{\partial r} \frac{\partial^{2} F}{\partial r^{2}} \\
-2 c \frac{\partial w}{\partial t}-\frac{1}{1-\nu} \nabla^{2} M_{T} \\
\nabla^{4} F=-\frac{E h}{r} \frac{\partial^{2} w}{\partial r^{2}} \frac{\partial w}{\partial r}-\frac{1}{h} \nabla^{2} N_{T}
\end{gathered}
$$

where

$$
\begin{aligned}
& N_{T}=E \alpha \int_{-\frac{1}{2} h}^{\frac{1}{2} h} T(r, z) d z \\
& M_{T}=E \alpha \int_{-\frac{1}{2} h}^{\frac{1}{2} h} T(r, z) z d z
\end{aligned}
$$

and $D=\frac{E h^{3}}{12\left(1-\nu^{2}\right)}$. Here, $w(r, t)$ is the plate transverse displacement, $F(r, t)$ is the stress function, $e$ is the dilatational strain due to the thermal effect, $T$ is the temperature distribution, $\rho$ is the material density, $h$ is the plate thickness, $c_{p}$ is the heat capacity coefficient at constant pressure, $E$ is the modulus of elasticity, $\alpha$ is the coefficient of thermal expansion, $Q$ is the heat flux, and $\nu$ is Poisson's ratio. We consider the case in which the plate is exposed to a uniformly distributed external heat flux $Q=4 k q \cos \omega t$ and the temperature is kept constant at the plate edge at $T_{0}$. The temperature distribution is governed by the heat conduction Eq. (1). For boundary conditions, we consider a simply supported plate with no restrictions on its in-plane displacement; that is , movable boundary condition. This case is important in the structural mechanics of planar bodies, such as beams and plates Vinson [22]. In this case, the thermal stresses in the plate are self-equilibrating and the radial force vanishes at the outer edge. Therefore, the boundary conditions are

$$
F<\infty \text { and } w<\infty \text { at } r=0
$$

$$
\begin{aligned}
& \frac{\partial F}{\partial r}=0 \text { at } r=R \\
& w=0 \text { and } \frac{\partial^{2} w}{\partial r^{2}}+\frac{\nu}{r} \frac{\partial w}{\partial r}=0 \text { at } r=R
\end{aligned}
$$

where $R$ is the radius of the plate.

We introduce nondimensional variables, denoted by asterisks, defined as follows:

$$
\begin{aligned}
r & =R r^{*}, \quad t=R^{2}\left(\frac{\rho h}{D}\right)^{1 / 2} t^{*}, \\
T & =T_{0} T^{*}, \quad w=\frac{h^{2}}{R} w^{*}, \\
c & =\frac{12\left(1-\nu^{2}\right)}{R^{4}}\left(\rho h^{5} D\right)^{1 / 2} c^{*}, \\
F & =\frac{E h^{5}}{R^{2}} F^{*}, \quad q=\frac{h^{5} q^{*}}{4 \alpha R^{6}}
\end{aligned}
$$

Substituting Eq. (9) into Eq. (1) and dropping the asterisks, we obtain

$$
\nabla^{2} T+\frac{Q R^{2}}{k T_{0}}=\Gamma_{1} \frac{\partial T}{\partial t}+\Gamma_{2} \frac{\partial e}{\partial t}
$$

where

$$
\Gamma_{1}=\frac{\rho c_{p}}{k}\left(\frac{D}{\rho h}\right)^{1 / 2}
$$

and 
Table 1

Thermal and mechanical properties of used materials

\begin{tabular}{lccccccl}
\hline Material & $\rho\left(\mathrm{Kg} / \mathrm{m}^{3}\right)$ & $E(\mathrm{GPa})$ & $k(\mathrm{~W} / \mathrm{m} . \mathrm{K})$ & $c_{p}(\mathrm{~J} / \mathrm{Kg} . \mathrm{K})$ & $\alpha$ & $\Gamma 1$ & $\Gamma 2$ \\
\hline Al & 2702 & 70 & 237 & 903 & $2410^{-6}$ & $15.8610^{6} \mathrm{~h}$ & $27.310^{6} \mathrm{~h}$ \\
Steel & 7800 & 207 & 60 & 430 & $1210^{-6}$ & $97.4310^{6} \mathrm{~h}$ & $161.3410^{6} \mathrm{~h}$ \\
SiNi & 2330 & 100 & 148 & 712 & $2.910^{-6}$ & $22.2410^{6} \mathrm{~h}$ & $9.7210^{6} \mathrm{~h}$ \\
\hline
\end{tabular}

$$
\Gamma_{2}=\frac{E \alpha}{(1-2 v)} \frac{1}{k}\left(\frac{D}{\rho h}\right)^{1 / 2}
$$

The last two terms on the right-hand side of Eq. (10) represent the diffusion of heat and thermoelastic coupling [8].

Usually, the materials used in plates for mechanical and MEMS applications are Steel, Aluminium (Al), and Silicon Nitride (SiNi). Using the available mechanical and thermal properties for these materials listed in Table 1, we calculate and list the results of the nondimensional coefficients $\Gamma_{1}$ and $\Gamma_{2}$ in Table 1. For thin plates, it follows from Eq. (10) and Table 1 that the thermal diffusion and thermoelastic coupling terms are negligible because $\Gamma_{1}$ and $\Gamma_{2}$ are very large. Hence, Eq. (1) is reduced to

$$
k \nabla^{2} T+4 k q \cos \omega t=0
$$

Solving Eq. (11) subject to the boundary conditions $T=T_{0}$ at $r=R$ and $T<\infty$ at $r=0$, we have

$$
T=T_{0}+\left(R^{2}-r^{2}\right) q \cos \omega t
$$

Substituting Eq. (12) into Eqs (4) and (5) yields

$$
\begin{aligned}
& N_{T}=E \alpha \int_{-\frac{1}{2} h}^{\frac{1}{2} h} T(r) d z=E \alpha h T(r) \\
& M_{T}=E \alpha \int_{-\frac{1}{2} h}^{\frac{1}{2} h} T(r) z d z=0
\end{aligned}
$$

Now, substituting Eqs (9), (13) and (14) into Eqs (2) and (3) and dropping the asterisks, we obtain

$$
\begin{gathered}
\frac{\partial^{2} w}{\partial t^{2}}+\nabla^{4} w \\
=\epsilon\left[\frac{1}{r} \frac{\partial^{2} w}{\partial r^{2}} \frac{\partial F}{\partial r}+\frac{1}{r} \frac{\partial w}{\partial r} \frac{\partial^{2} F}{\partial r^{2}}-2 c \frac{\partial w}{\partial t}\right] \\
\nabla^{4} F=-\frac{1}{2 r} \frac{\partial}{\partial r}\left(\frac{\partial w}{\partial r}\right)^{2}+q \cos \omega t \\
w<\infty \text { and } F<\infty \text { at } r=0 \\
\frac{\partial F}{\partial r}=0 \text { at } r=1 \\
w=0 \text { and } \frac{\partial^{2} w}{\partial r^{2}}+\frac{\nu}{r} \frac{\partial w}{\partial r}=0 \text { at } r=1 \\
\text { where } \epsilon=\frac{12\left(1-\nu^{2}\right) h^{2}}{R^{2}} .
\end{gathered}
$$

\section{Perturbation solution}

We seek a first-order uniform expansion of the solution of Eqs (15)-(19) in the form [13]

$$
\begin{aligned}
w(r, t ; \epsilon)= & w_{0}\left(r, t_{0}, t_{1}\right) \\
& +\epsilon w_{1}\left(r, t_{0}, t_{1}\right)+\cdots \\
F(r, t ; \epsilon)= & F_{0}\left(r, t_{0}, t_{1}\right)+\cdots
\end{aligned}
$$

where $t_{0}=t$ and $t_{1}=\epsilon t$. Hence, the time derivative is transformed into

$$
\frac{d}{d t}=D_{0}+\epsilon D_{1}+\cdots
$$

where $D_{n}=\partial / \partial t_{n}$. Substituting Eqs (20)-(22) into Eqs (15)-(19) and equating coefficients of like powers of $\epsilon$, we obtain

Order $\epsilon^{0}$

$$
\begin{aligned}
& D_{0}^{2} w_{0}+\nabla^{4} w_{0}=0 \\
& \nabla^{4} F_{0}=-\frac{1}{2 r} \frac{\partial}{\partial r}\left(\frac{\partial w_{0}}{\partial r}\right)^{2}+q \cos \omega T_{0} \\
& w_{0}<\infty \text { and } F_{0}<\infty \text { at } r=0 \\
& F_{0}^{\prime}=0 \text { at } r=1 \\
& w_{0}=0 \text { and } w_{0}^{\prime \prime}+\nu w_{0}^{\prime}=0 \text { at } r=1
\end{aligned}
$$

Order $\epsilon$

$$
\begin{aligned}
& D_{0}^{2} w_{1}+\nabla^{4} w_{1} \\
= & -2 D_{0} D_{1} w_{0}+\frac{1}{r} \frac{\partial^{2} w_{0}}{\partial r^{2}} \frac{\partial F_{0}}{\partial r} \\
& +\frac{1}{r} \frac{\partial w_{0}}{\partial r} \frac{\partial^{2} F_{0}}{\partial r^{2}}-2 c D_{0} w_{0} \\
w_{1} & <\infty \text { at } r=0 \\
w_{1}= & 0 \text { and } w_{1}^{\prime \prime}+\nu w_{1}^{\prime}=0 \text { at } r=1
\end{aligned}
$$

where the prime indicates the partial derivative with respect to $r$. 
The solution of Eqs (23), (25) and (27) can be expressed as

$$
\begin{aligned}
& w_{0}= \\
& \sum_{n=1}^{\infty}\left[A_{n}\left(t_{1}\right) e^{i \omega_{n} t_{0}}+\bar{A}_{n}\left(t_{1}\right) e^{-i \omega_{n} t_{0}}\right] \phi_{n}(r)
\end{aligned}
$$

where

$$
\begin{aligned}
\phi_{n}(r)= & k_{n}\left[I_{0}\left(\sqrt{\omega_{n}}\right) J_{0}\left(\sqrt{\omega_{n}} r\right)\right. \\
& \left.-J_{0}\left(\sqrt{\omega_{n}}\right) I_{0}\left(\sqrt{\omega_{n}} r\right)\right]
\end{aligned}
$$

the $\omega_{n}$ are the roots of

$$
\frac{J_{1}\left(\sqrt{\omega_{n}}\right)}{J_{0}\left(\sqrt{\omega_{n}}\right)}+\frac{I_{1}\left(\sqrt{\omega_{n}}\right)}{I_{0}\left(\sqrt{\omega_{n}}\right)}=\frac{2 \sqrt{\omega_{n}}}{1-\nu}
$$

and the $k_{n}$ are chosen so that

$$
\int_{0}^{1} r \phi_{n}^{2}(r) d r=1
$$

The lowest five nondimensional frequencies for the case $\nu=0.3$ are $4.935,29.720,74.156,138.318$, and 222.215. Hence, the first mode is not involved in any internal resonance with any other symmetric mode. Moreover, this mode is not involved in an internal resonance with any asymmetric mode. In this paper, we consider the case of principal parametric resonance of the first mode. The analysis is also valid for any mode that is not involved in an internal resonance with other modes. For asymmetric thermal loads, one-to-one internal resonance might be activated. This case is not considered in this paper.

Substituting Eq. (31) into Eq. (24) yields

$$
\begin{aligned}
\nabla^{4} F_{0}= & -\frac{1}{2 r} \sum_{m, n}\left[A_{m} A_{n} e^{i\left(\omega_{n}+\omega_{m}\right) t_{0}}\right. \\
& \left.+A_{n} \bar{A}_{m} e^{i\left(\omega_{n}-\omega_{m}\right) t_{0}}\right] \\
& \left(\phi_{n}^{\prime} \phi_{m}^{\prime}\right)^{\prime}+c c+q \cos \omega t_{0}
\end{aligned}
$$

The solution of Eq. (35) subject to the boundary conditions Eqs (25) and (26) can be expressed as

$$
\begin{aligned}
F_{0}= & \sum_{m, n}\left[A_{n} A_{m} e^{i\left(\omega_{n}+\omega_{m}\right) t_{0}}\right. \\
& \left.+A_{n} \bar{A}_{m} e^{i\left(\omega_{n}-\omega_{m}\right) t_{0}}\right] \psi_{n m}(r) \\
& +c c+b+\frac{1}{16}\left(r^{4}-2 r^{2}\right) q \cos \omega t_{0}
\end{aligned}
$$

where $b$ is a constant and

$$
\nabla^{4} \psi_{n m}=-\frac{1}{2 r}\left(\phi_{n}^{\prime} \phi_{m}^{\prime}\right)^{\prime}
$$

$$
\begin{aligned}
& \psi_{n m}(0)<\infty \\
& \psi_{n m}^{\prime}=0 \text { at } r=1
\end{aligned}
$$

Multiplying Eq. (37) with $r$ and integrating the result, we have

$$
\left(r \frac{d^{2}}{d r^{2}}+\frac{d}{d r}-\frac{1}{r}\right) \frac{d \psi_{n m}}{d r}=-\frac{1}{2} \phi_{n}^{\prime} \phi_{m}^{\prime}
$$

where the constant of integration is incorporated in Eq. (36). Because

$$
\begin{aligned}
& \left(r^{2} \frac{d^{2}}{d r^{2}}+r \frac{d}{d r}-1\right) J_{1}\left(\xi_{k} r\right) \\
= & -\xi_{k}^{2} r^{2} J_{1}\left(\xi_{k} r\right)
\end{aligned}
$$

we express $\psi_{n m}^{\prime}$ as

$$
\psi_{n m}^{\prime}(r)=\sum_{k=1}^{\infty} b_{n m k} J_{1}\left(\xi_{k} r\right)
$$

Using the boundary condition Eq. (39), we find that the $\xi_{m}$ are the roots of

$$
J_{1}\left(\xi_{k}\right)=0
$$

The lowest ten roots are $0,3.832,7.016,13.324$, 19.616, 22.760, 25.904, 29.047, 32.190, and 35.332. Substituting Eq. (42) into Eq. (40) and using Eq. (41), we obtain

$$
\begin{aligned}
& \sum_{k=1}^{\infty} b_{n m k} \\
& {\left[r J_{1}^{\prime \prime}\left(\xi_{k} r\right)+J_{1}^{\prime}\left(\xi_{k} r\right)-\frac{1}{r} J_{1}\left(\xi_{k} r\right)\right] } \\
= & \sum_{k=1}^{\infty} b_{n m k} \xi_{k}^{2} r J_{1}\left(\xi_{k} r\right) \\
= & -\frac{1}{2} \phi_{n}^{\prime} \phi_{m}^{\prime}
\end{aligned}
$$

Multiplying Eq. (44) with $J_{1}\left(\xi_{s} r\right)$ and integrating the result with respect to $r$ from $r=0$ to 1 , we have

$$
\begin{aligned}
b_{n m s}= & \int_{0}^{1} J_{1}\left(\xi_{s} r\right) \phi_{n}^{\prime}(r) \phi_{m}^{\prime}(r) d r / \\
& {\left[2 \xi_{s}^{2} \int_{0}^{1} r J_{1}^{2}\left(\xi_{s} r\right) d r\right] }
\end{aligned}
$$

Substituting Eqs (31) and (36) into Eq. (28) yields

$$
\begin{aligned}
& D_{0}^{2} w_{1}+\nabla^{4} w_{1} \\
= & -2 i \sum_{n} \omega_{n}\left(A_{n}^{\prime}+c A_{n}\right) \phi_{n} e^{i \omega_{n} t_{0}}
\end{aligned}
$$




$$
\begin{aligned}
& +\frac{1}{8} q e^{i \omega t_{0}} \sum_{n}\left[\left(r^{2}-1\right) \phi_{n}^{\prime \prime}+\frac{3 r^{2}-1}{r} \phi_{n}^{\prime}\right] \\
& {\left[A_{n} e^{i \omega_{n} t_{0}}+\bar{A}_{n} e^{-i \omega_{n} t_{0}}\right]} \\
& +\frac{1}{r} \sum_{n, m, s}\left(\phi_{s}^{\prime} \psi_{n m}^{\prime}\right)^{\prime} \\
& {\left[A_{s} A_{n} A_{m} e^{i\left(\omega_{n}+\omega_{m}+\omega_{s}\right) t_{0}}\right.} \\
& +A_{s} A_{n} \bar{A}_{m} e^{i\left(\omega_{s}+\omega_{n}-\omega_{m}\right) t_{0}} \\
& +A_{n} A_{m} \bar{A}_{s} e^{i\left(\omega_{n}+\omega_{m}-\omega_{s}\right) t_{0}} \\
& \left.+A_{n} \bar{A}_{m} \bar{A}_{s} e^{i\left(\omega_{n}-\omega_{m}-\omega_{s}\right) t_{0}}\right]+c c
\end{aligned}
$$

Next, we consider the case of principal parametric resonance of the $j$ th mode and assume that it is not involved in an internal resonance with any other mode. To describe the nearness of the resonance, we introduce a detuning parameter $\sigma$ defined by

$$
\omega=2 \omega_{j}+\epsilon \sigma
$$

Substituting Eq. (47) into Eq. (46) yields

$$
\begin{aligned}
& D^{2} w_{1}+\nabla^{4} w_{1} \\
= & -2 i \sum_{n} \omega_{n}\left(A_{n}^{\prime}+c A_{n}\right) \phi_{n} e^{i \omega_{n} t_{0}} \\
& +\frac{1}{8} q \sum_{n}\left[\left(r^{2}-1\right) \phi_{n}^{\prime \prime}+\frac{3 r^{2}-1}{r} \phi_{n}^{\prime}\right] \\
& {\left[A_{n} e^{i\left(\omega_{n}+2 \omega_{j}\right) t_{0}+i \sigma t_{1}}\right.} \\
& \left.+\bar{A}_{n} e^{i\left(2 \omega_{j}-\omega_{n}\right) t_{0}+i \sigma t_{1}}\right] \\
& +\frac{1}{r} \sum_{n, m, s}\left(\phi_{s}^{\prime} \psi_{n m}^{\prime}\right)^{\prime} \\
& {\left[A_{s} A_{n} A_{m} e^{i\left(\omega_{n}+\omega_{m}+\omega_{s}\right) t_{0}}\right.} \\
& +A_{s} A_{n} \bar{A}_{m} e^{i\left(\omega_{s}+\omega_{n}-\omega_{m}\right) t_{0}} \\
& +A_{n} A_{m} \bar{A}_{s} e^{i\left(\omega_{n}+\omega_{m}-\omega_{s}\right) t_{0}} \\
& \left.+A_{n} \bar{A}_{m} \bar{A}_{s} e^{i\left(\omega_{n}-\omega_{m}-\omega_{s}\right) t_{0}}\right]+c c
\end{aligned}
$$

In the presence of damping, all modes that are not directly excited or indirectly excited by an internal resonance decay with time, and hence the long-time response consists of the $j$ th mode only. Our choice here will be for the first mode. Because the homogeneous problem consisting of Eqs (48), (29) and (30) has nontrivial solutions, the nonhomogeneous problem has a solution only if a solvability condition is satisfied. Be- cause the homogeneous problem is self-adjoint [14,15], the solvability condition demands that the right-hand of Eq. (48) be orthogonal to every solution of the homogeneous problem. Multiplying the right-hand side of Eq. (48) with $r \phi_{j}(r) e^{-i \omega_{j} t_{0}}$, integrating the result from $r=0$ to $r=1$, and setting the result equal to zero, we obtain

$$
\begin{aligned}
& 2 i \omega_{j}\left(A_{j}^{\prime}+\mu_{j} A_{j}\right)+\alpha_{e} A_{j}^{2} \bar{A}_{j} \\
+ & q_{e} \bar{A}_{j} e^{i \sigma t_{1}}=0
\end{aligned}
$$

where

$$
\begin{aligned}
\alpha_{e} & =\int_{0}^{1} \phi_{j}\left(\phi_{j}^{\prime} \psi_{j j}^{\prime}\right)^{\prime} d r \\
& =-\int_{0}^{1} \phi_{j}^{\prime 2} \psi_{j j}^{\prime} d r \\
q_{e} & =\frac{1}{8} q \int_{0}^{1}\left(\left(r^{3}-r\right) \phi_{j}^{\prime}\right)^{\prime} \phi_{j} d r \\
& =-\frac{1}{8} q \int_{0}^{1}\left(r^{3}-r\right) \phi_{j}^{\prime 2} d r
\end{aligned}
$$

\section{Numerical results}

To investigate the solutions of Eq. (49), we introduce the polar transformation

$$
A_{j}=\frac{1}{2} a_{j} e^{i\left(\beta+\frac{1}{2} \sigma t_{1}\right)}
$$

separate real and imaginary parts, and obtain

$$
\begin{gathered}
a_{j}^{\prime}=-\mu_{j} a_{j}+\frac{q_{e}}{2 \omega_{j}} a_{j} \sin 2 \beta \\
a_{j} \beta_{j}^{\prime}=-\frac{1}{2} \sigma a_{j}+\frac{\alpha_{e}}{8 \omega_{j}} a_{j}^{3} \\
+\frac{q_{e}}{2 \omega_{j}} a_{j} \cos 2 \beta
\end{gathered}
$$

The equilibrium solutions of Eqs (53) and (54) correspond to periodic motions of the plate. The equilibrium solutions are given by

$$
\begin{gathered}
\mu_{j} a_{j}=\frac{q_{e}}{2 \omega_{j}} a_{j} \sin 2 \beta \\
\frac{1}{2} \sigma a_{j}-\frac{\alpha_{e}}{8 \omega_{j}} a_{j}^{3}=\frac{q_{e}}{2 \omega_{j}} a_{j} \cos 2 \beta
\end{gathered}
$$

There are two possibilities: either $a_{j}=0$ or $a_{j} \neq 0$. In the latter case, eliminating $\beta$ from Eqs (55) and (56) yields the frequency-response equation 


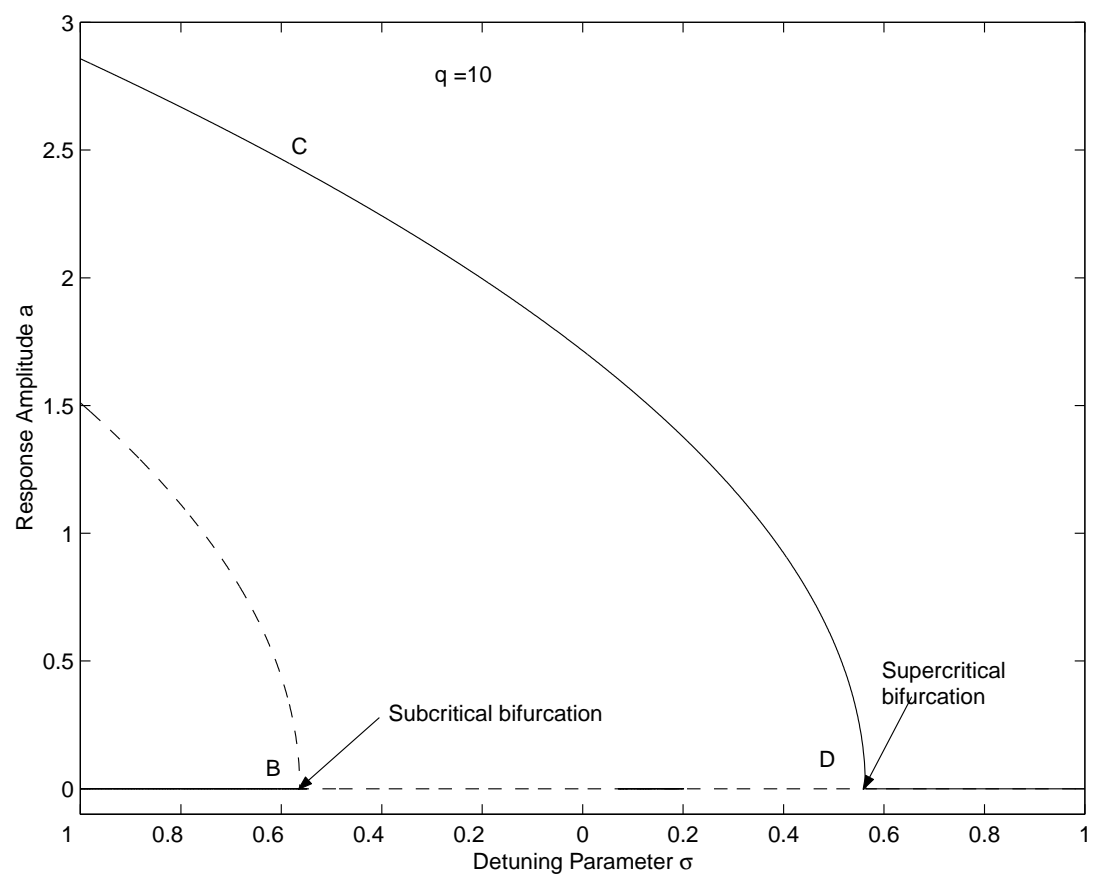

Fig. 2. Variation of the steady-state response amplitude with the detuning parameter.

$$
\mu_{j}^{2}+\left(\frac{1}{2} \sigma-\frac{\alpha_{e}}{8 \omega_{j}} a_{j}^{2}\right)^{2}=\frac{q_{e}^{2}}{4 \omega_{j}^{2}}
$$

or

$$
a_{j}^{2}=\frac{8 \omega_{j}}{\alpha_{e}}\left[\frac{1}{2} \sigma \pm \sqrt{\frac{q_{e}^{2}}{4 \omega_{j}^{2}}-\mu_{j}^{2}}\right]
$$

Equation (58) represents the relation between the response amplitude and the forcing amplitude and detuning parameter, the latter are considered the control parameters. Next, we present results for the first mode. The $b$ values in Eq. (45) are: 1.24252, $-0.144995,-0.0272522,-0.0102221,0.00702803$, $-0.00507591, \quad 0.00380701, \quad-0.00294182$, and 0.00232898 . This leads to $\alpha_{e}=-3.77941$. Also, we assume that $\mu=0.01$ and consider nondimensional values of $q$ ranging from 1 to 30. In Fig. 2, the frequency -response curve is shown for $q=10$. The curve is bent to the left, indicating a softening-type nonlinearity. The bending of the frequency-response curves leads to multivalued amplitudes and hence to jumps. To illustrate the jump phenomenon, let us suppose that an experiment is conducted where the excitation amplitude is kept constant while the excitation frequency is varied very slowly. We start from a frequency far below the natural frequency (i.e., is negatively large) and increase it. We chose the initial conditions so that the response amplitude is trivial. As $\sigma$ is increased, the response amplitude remains trivial till point $\mathrm{B}$ is reached, where the response amplitude experiences a sudden jump up to point $\mathrm{C}$. Point $\mathrm{B}$ is a subcritical or reverse pitchfork bifurcation. The response amplitude decreases as the frequency is further increased till point $\mathrm{D}$ is reached, where it becomes trivial again. Point D is a supercritical pitchfork bifurcation.

Starting at point $\mathrm{C}$ and sweeping in the reverse direction, we note that, to the first-order approximation, the response amplitude increases without bound as the frequency decreases. In reality, the stable and unstable branches merge to produce a saddle-node bifurcation. Decreasing below this point results in a jump down of the response amplitude to the trivial solution. Between point $\mathrm{B}$ and the saddle-node bifurcation, there are three possible solutions: the trivial solution, which is stable, and two nontrivial solutions, the larger of which is stable and the smaller is unstable. In this interval, the response may be trivial or nontrivial, depending on the initial conditions.

In Fig. 3, a force-response curve is generated when the system is positively detuned by 0.5 . Starting from a forcing amplitude corresponding to point $\mathrm{E}$ and increasing it results in a monotonic increase in the response amplitude. Point $\mathrm{E}$ is a supercritical pitchfork bifurcation. For any excitation amplitude above point $\mathrm{E}$, the response amplitude settles on the branch $\mathrm{EB}$, irrespective of the initial conditions. Sweeping $q$ 


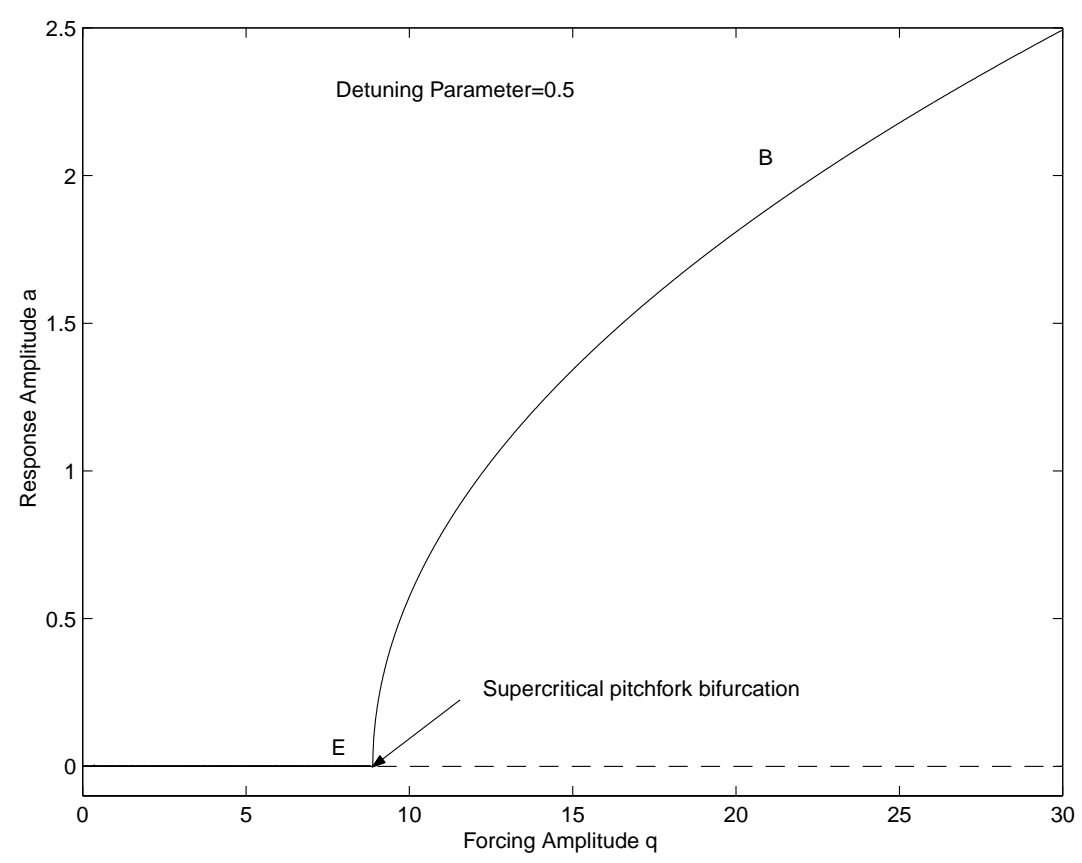

Fig. 3. Variation of the steady-state response amplitude with the forcing amplitude for positive detuning parameter.

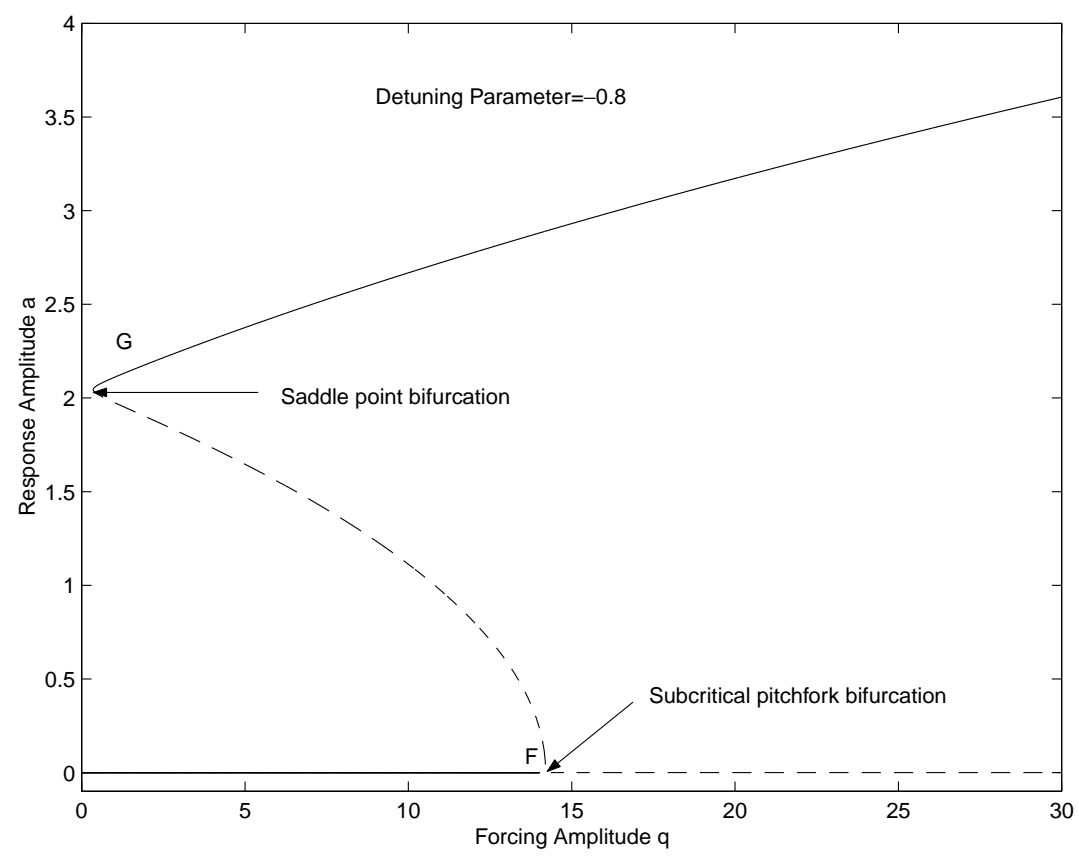

Fig. 4. Variation of the steady-state response amplitude with the forcing amplitude for negative detuning parameter.

down, one finds that the response amplitude follows the branch BE. Decreasing $q$ below point E results in a smooth transition of the response from a nontrivial to a trivial response. Increasing the value of the detuning parameter results in a shift of the critical point $E$ to the right.

We show in Fig. 4 the force-response curve for the case of a negative detuning of -0.8 . In contrast with the preceding case, there are multivalued responses. Point $F$ is a subcritical pitchfork bifurcation and point $G$ 
is a saddle-node bifurcation. Between these bifurcations, the response may be trivial or nontrivial depending on the initial conditions. Whereas the linear theory predicts stable trivial responses for forcing amplitudes below point $\mathrm{F}$ and unbounded responses above it, the nonlinear analysis predicts instabilities of the trivial response between points $\mathrm{F}$ and $\mathrm{G}$, a subcritical instability. Moreover, the nonlinearity puts a cap on the response amplitude.

Sweeping $q$ in the forward direction, one finds that the response amplitude jumps up beyond point F. On the other hand, sweeping $q$ in the reverse direction results in a jump to the trivial response beyond point $\mathrm{G}$.

\section{Summary}

Thermally induced vibrations are of concern in the design of many structural elements. Most of the previous research on thermally induced vibrations in circular plates has been limited to solving for small deflections. Moreover, the works that deal with large deflections were based on the Berger's approximation. In this paper, we investigate the nonlinear response of a circular plate to a thermal loading consisting of a steady component and a sinusoidal component. We base the analysis on the dynamic analogue of the von Karman equations. We first formulate the heat conduction problem and solve it for the temperature distribution. Then, we substitute the resulting distribution into the equations describing the displacement. We use the method of multiple scales to determine a first-order approximation of the plate response in the case of principal parametric resonance of an axisymmetric mode, which is not involved in an internal resonance with any other mode, and obtain two first-order nonlinear ordinary-differential equations governing the amplitude and phase of the response. We use these modulation equations to generate frequency-and forceresponse curves. The numerical results show that the heat variation can lead to large-amplitude responses, including multivalued responses and jumps.

\section{Acknowledgment}

This work was supported by the Air Force Office of Scientific Research under Grant No. F49620-98-10393.

\section{References}

[1] G.A. Altay and M.C. Dokmeci, Karman-Mindlin plate equations for thermoelastic vibrations of temperature dependent materials, ARI 50 (1997), 110-126.

[2] F. Buckens, Vibrations in a thermally stressed thin plate, Journal of Thermal Stresses 2 (1979), 367-385.

[3] B.A. Boley and J.H. Weiner, Theory of thermal stresses, Wiley, 1960.

[4] W.P. Chang and S.M. Wan, Thermomechanically coupled nonLinear vibration of plates, International Journal of Non-Linear Mechanics 21(5) (1986), 375-389.

[5] B.E. Gatewood, Thermal stresses, McGraw-Hill, 1957.

[6] C. Gehin, C. Barthod and Y. Teisseyre, Design and characterisation of ane force resonant sensor, Sensors and Actuators A: Physical 84 (2000), 65-69.

[7] R. R. Heldenfels and W. M. Roberts, Experimental and theoretical determination of thermal stresses in a flat plate, NACA Technical Note 2769, 1952.

[8] R.B. Hetnarski, Thermal stresses II, North-Holland, 1987.

[9] R. Heuer, H. Irschik and U. Pichler, Free large vibration of thermally buckled plates: a unifying formulation, in: Eighth Conference on Nonlinear Vibrations, Stability, and Dynamics of Structures, Blacksburg, VA, 2000.

[10] R. Jones, J. Mazumdar and Y.K. Cheung, Vibration and buckling of plates at elevated temperatures, International Journal of Solids and Structures 16 (1980), 61-70.

[11] L. Librescu and W. Lin, Non-linear response of laminated plates and shells to thermomechanical loading: implications of violation of interlaminar shear traction continuity requirement, International Journal of Solids and Structures 36 (1999), 4111-4147.

[12] K. Marguerre, 1935, Thermo-elastische platten-gleich-ungen, Zeitschrift fur Angewandte Mathematik und Mechanik 15 (1935), 369-372.

[13] A.H. Nayfeh, Introduction to perturbation techniques, Wiley, 1981.

[14] A.H. Nayfeh, Nonlinear interactions, Wiley, 2000.

[15] A.H. Nayfeh and D.T. Mook, Nonlinear oscillations, Wiley, 1979.

[16] M.C. Pal, Large deflections of heated circular plates, Acta Mechanica 8 (1969), 82-103.

[17] M.C. Pal, Large amplitude free vibration of circular plates subjected to aerodynamic heating, International Journal of Solids and Structures 6 (1970), 301-313.

[18] M.C. Pal, Static and dynamic non-linear behaviour of heated orthotropic circular plates, International Journal of NonLinear Mechanics 8 (1973), 489-504.

[19] R. Puers, A. Cozma and D. De Bruyker, On the mechanisms in thermally actuated composite diaphragm, Sensors and Actuators A 67 (1998), 13-17.

[20] I.S. Sokolnikoff and E.S. Sokolnikoff, Thermal stresses in elastic plates, Transactions of The American Mathematical Society 45 (1939), 235-255.

[21] M. Sunakawa, Influence of temperature changes and large amplitude on free flexural vibration of rectangular elastic plates, Transactions of JSME 30 (1964), 558-566.

[22] J.R. Vinson, The behavior of thin walled structures, Kluwer, 1989. 

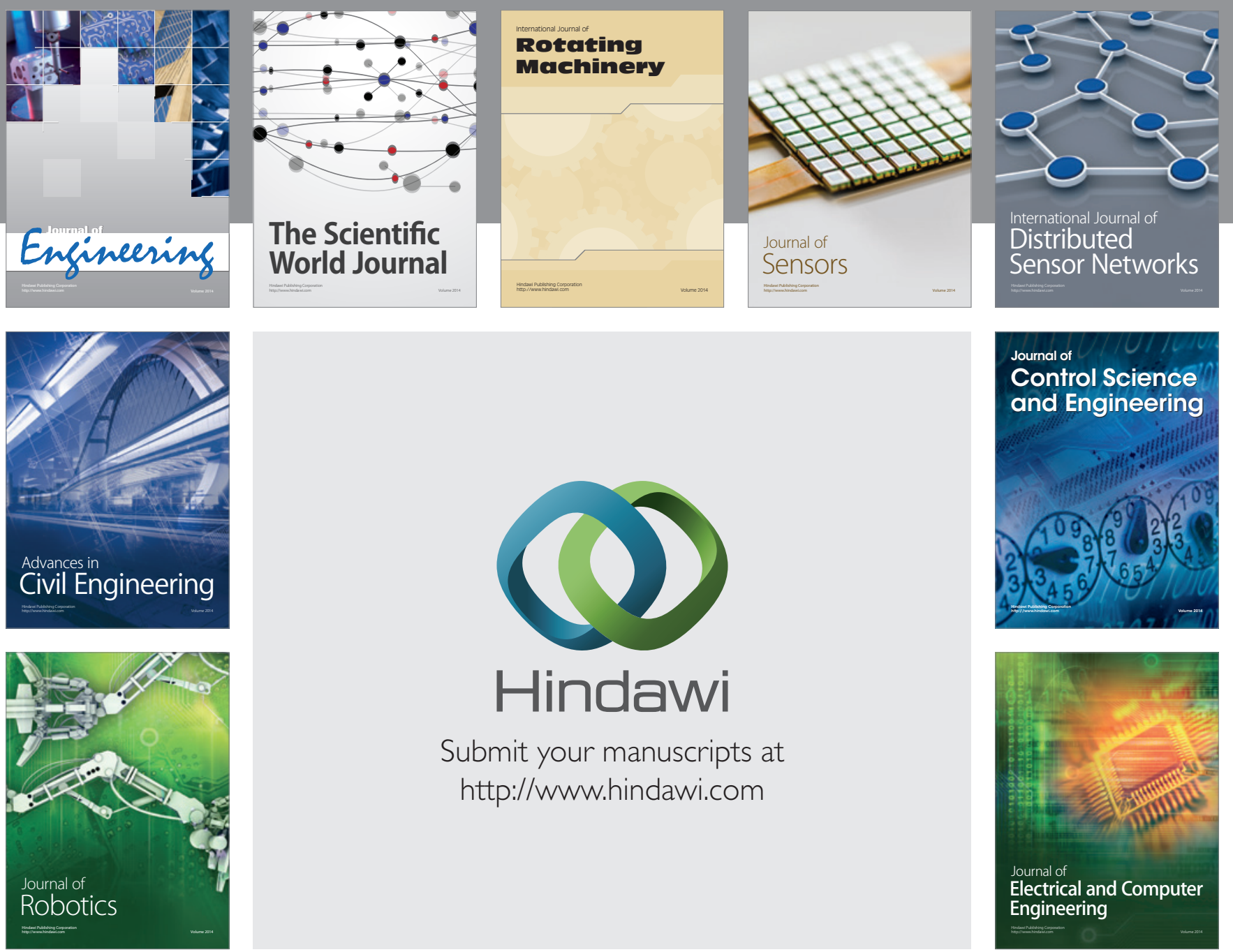

Submit your manuscripts at

http://www.hindawi.com
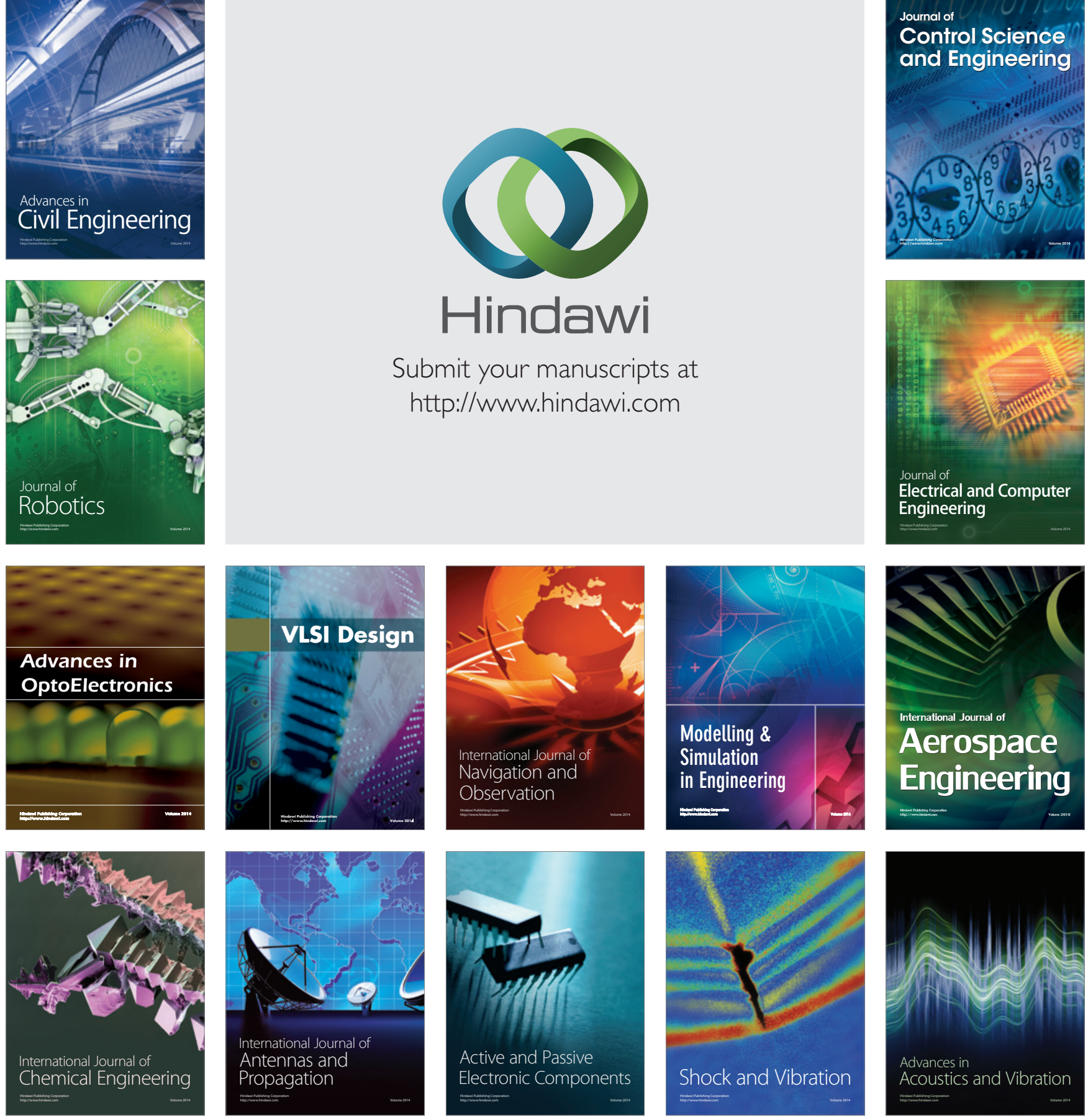